\title{
Article \\ A Comparison of Laboratory Simulation Methods of Iron Contamination for FCC Catalysts
}

\author{
Yitao Liao ${ }^{1,2}$, Tao Liu ${ }^{2}$, Huihui Zhao ${ }^{2}$ and Xionghou Gao ${ }^{2, *}$ \\ 1 College of Chemistry and Chemical Engineering, Northwest Normal University, Lanzhou 730070, China; \\ liaoyitao@petrochina.com.cn \\ 2 Lanzhou Petrochemical Research Center, Petrochemical Research Institute, PetroChina, Lanzhou 730060, \\ China; liutao5@petrochina.com.cn (T.L.); zhaohuihui3@petrochina.com.cn (H.Z.) \\ * Correspondence: gaoxionghou@petrochina.com.cn; Tel.: +86-010-8016-5606
}

check for

updates

Citation: Liao, Y.; Liu, T.; Zhao, H.;

Gao, X. A Comparison of Laboratory Simulation Methods of Iron Contamination for FCC Catalysts. Catalysts 2021, 11, 104. https:// doi.org/10.3390/catal11010104

Received: 11 December 2020 Accepted: 7 January 2021

Published: 14 January 2021

Publisher's Note: MDPI stays neutral with regard to jurisdictional clai$\mathrm{ms}$ in published maps and institutional affiliations.

Copyright: (C) 2021 by the authors. Licensee MDPI, Basel, Switzerland. This article is an open access article distributed under the terms and conditions of the Creative Commons Attribution (CC BY) license (https:// creativecommons.org/licenses/by/ $4.0 /)$.

\begin{abstract}
Two different methods of simulating iron contamination in a laboratory were studied. The catalysts were characterized using $X$-ray diffraction, $\mathrm{N}_{2}$ adsorption-desorption, and SEM-EDS. The catalyst performance was evaluated using an advanced cracking evaluation device. It was found that iron was evenly distributed in the catalyst prepared using the Mitchell impregnation method and no obvious iron nodules were found on the surface of the catalyst. Iron on the impregnated catalyst led to a strong dehydrogenation capacity and a slight decrease in the conversion and bottoms selectivity. The studies also showed that iron was mainly in the range of $1-5 \mu \mathrm{m}$ from the edge of the catalyst prepared using the cycle deactivation method. Iron nodules could be easily observed on the surface of the catalyst. The retention of the surface structure in the alumina-rich areas and the collapse of the surface structure in the silica-rich areas resulted in a continuous nodule morphology, which was similar to the highly iron-contaminated equilibrium catalyst. Iron nodules on the cyclic-deactivated catalyst led to a significant decrease in conversion, an extremely high bottoms yield, and a small increase in the dehydrogenation capacity. The nodules and distribution of iron on the equilibrium catalyst could be better simulated by using the cyclic deactivation method.
\end{abstract}

Keywords: Mitchell impregnation; cyclic deactivation; iron contamination; iron nodules

\section{Introduction}

How to test a fluid catalytic cracking (FCC) catalyst's performance and metal tolerance in the laboratory and calibrate the results to simulate the industrial performance is an ongoing discussion. Another important aspect of catalyst testing is the preparation of the catalyst [1]. In evaluating a fluid cracking catalyst's performance in lab-scale testing, selecting the proper catalyst deactivation method is just as important as the testing itself [2].

When heavy metals are deposited on the catalyst, the physical and chemical properties of the catalyst will be affected by the distribution, valence state, and existing forms of the heavy metals. Therefore, it is difficult to accurately simulate the industrial heavy-metalpolluted equilibrium catalyst by selecting the laboratory deactivation protocols. Recently, due to the relatively low supply of light and low-sulfur crude oil, which has a relatively high market price, refiners are obliged to process increasing quantities of heavy crude by cracking them into lighter distillates [3,4]. With the increasing trend of heavy and poor-quality FCC feedstocks, the quantity and species of heavy metal deposition, such as $\mathrm{Ni}[5,6], \mathrm{V}[7,8], \mathrm{Ca}[9,10], \mathrm{Na}[11]$, and especially iron [12], on the catalyst have gradually increased. Iron deactivation effects can be subdivided into the following two classifications: (1) iron nodules may affect the catalyst's apparent bulk density (ABD) and fluidization [13] and (2) increasing the amount of iron will cause a loss of activity and bottoms cracking, as well as increased SOx emissions and coke on the regenerated catalyst (CRC) in partial burn units [14]. 
Multiple kinds of heavy metals deposition on a catalyst increase the difficulty of selecting a deactivation protocol. The detrimental effects of nickel and vanadium that were studied using deactivation protocol simulations have been described elsewhere. Buurmans et al. [15] investigated the effect of nickel and vanadium deactivation on the structure and acidity of FCC catalyst particles by using steaming, cyclic deactivation, and the Mitchell impregnation method. Nguyen et al. [16] investigated the effect of hydrothermal conditions on the catalytic deactivation of an FCC catalyst by using a cyclic propylene steaming (CPS) method. Etim et al. $[17,18]$ investigated the role of nickel on a vanadiumpoisoned FCC catalyst and the effect of vanadium contamination on the framework and micropore structure by using the Mitchell impregnation method. However, few studies on the simulation and catalytic performance of iron contamination have been reported. Nobody has successfully simulated the iron nodules observed on the surface of an ironcontaminated equilibrium catalyst in the laboratory. If there is no accurate method for simulating iron deactivation, the research on the impact of iron contamination and the development of iron tolerance technology in the laboratory will not have real and reliable theoretical support, and sometimes wrong conclusions may be obtained. Therefore, in order to understand the real effect of iron on the catalyst, it is necessary to establish an accurate and effective simulation method of iron deactivation.

Laboratory catalyst deactivation protocols are used in order to simulate long-term commercial catalyst deactivation in an accelerated way [19]. A traditional way was proposed using the Mitchell impregnation method, which involves the impregnation of the catalyst with metal solutions, followed by calcination and a steam treatment [20]. An advanced method was to use the cyclic deactivation (CD) method, which has been described elsewhere. By using the CD method, Psarras et al. [21] investigated the accessibility effect on the irreversible deactivation of FCC catalysts. Almas et al. [22] studied the transformations of FCC catalysts and carbonaceous deposits during repeated reaction-regeneration cycles. Rainer et al. [23] investigated the Akzo Accessibility Index (AAI) of iron-contaminated catalysts under different conditions. One CD section consisted of repeated cycles of cracking, stripping, and regeneration treatment. The deactivation of the FCC catalyst in a CD unit is a simulation of the deactivation processes in a commercial plant.

In this work, a commercial catalyst (CAT-BASE) was characterized and tested in an advanced cracking evaluation (ACE) unit following cyclic deactivation (CAT-CD) and Mitchell impregnation with different iron species (CAT-MM1 using iron chloride and CAT-MM2 using iron naphthenate) in order to obtain a more direct comparison of the two laboratory methods regarding an iron-contaminated equilibrium catalyst simulation. Finally, the nodules, iron distribution, and the performance of the iron contaminated equilibrium catalyst could be better simulated by using the cyclic deactivation method.

\section{Results}

\subsection{Iron Deposited on the Catalyst Using Different Methods}

In order to study the effect of different contamination methods on the properties and catalytic performance of an iron-contaminated catalyst, three catalysts based on CATBASE were prepared by using iron chloride and iron naphthenate as precursors. An ironcontaminated equilibrium catalyst (E-cat) was selected as the target catalyst for different deactivation methods. CAT-BASE and E-cat were collected from the industrial plant of Dalian West Pacific Petrochemical Co., Ltd. (WEPEC, Dalian, China). The metal contents of the catalysts were obtained using XRF. The properties of the catalysts are shown in Table 1.

There was a certain amount of iron $(1828 \mu \mathrm{g} / \mathrm{g})$ on CAT-BASE, which came from the kaolin used to prepare the catalyst but it had no poisoning effect on the performance of the catalyst [24]. Three different metal-depositing methods were used to load almost the same amount of iron onto the catalyst. Finally, the target iron deposition was 12,000 $\mu \mathrm{g} / \mathrm{g}$, where the actual iron deposition on CAT-MM1 was 11,480 $\mu \mathrm{g} / \mathrm{g}$ and that on CAT-MM2 was $11,440 \mu \mathrm{g} / \mathrm{g}$. A similar amount of iron $(11,690 \mu \mathrm{g} / \mathrm{g})$ was deposited onto CAT-CD. 
Table 1. Properties of different catalysts.

\begin{tabular}{ccccccc}
\hline Catalysts & $\begin{array}{c}\boldsymbol{\omega}\left(\mathbf{N a}_{\mathbf{2}} \mathbf{O}\right) \\
\mathbf{( \% )}\end{array}$ & $\begin{array}{c}\boldsymbol{\omega}\left(\mathbf{A l}_{\mathbf{2}} \mathbf{O}_{\mathbf{3}}\right) \\
\mathbf{( \% )}\end{array}$ & $\begin{array}{c}\mathbf{S . A .} \\
\left(\mathbf{m}^{\mathbf{2}} \cdot \mathbf{g}^{-\mathbf{1})}\right.\end{array}$ & $\begin{array}{c}\mathbf{P . V} \cdot \text { total } \\
\left(\mathbf{c m}^{\mathbf{3}} \cdot \mathbf{g}^{-\mathbf{1}}\right)\end{array}$ & $\begin{array}{c}\text { Unit Cell } \\
\text { Size }(\mathbf{n m})\end{array}$ & $\begin{array}{c}\text { Fe on } \\
\text { Catalyst } \\
\left(\boldsymbol{\mu g} \cdot \mathbf{g}^{-\mathbf{1}} \mathbf{)}\right.\end{array}$ \\
\hline CAT-BASE & 0.16 & 48.6 & 269 & 0.42 & 2.462 & 1828 \\
CAT-MM1 & 0.16 & 48.6 & 149.4 & 0.21 & 2.440 & 13,308 \\
CAT-MM2 & 0.16 & 48.6 & 137.6 & 0.20 & 2.439 & 13,268 \\
CAT-CD & 0.16 & 48.6 & 122.5 & 0.16 & 2.436 & 13,518 \\
E-cat & 0.14 & 47.4 & 109.7 & 0.15 & 2.437 & 10,471 \\
\hline
\end{tabular}

S.A.: Surface Area; P.V.: Pore Volume; CAT-BASE: commercial catalyst, CAT-MM1: catalyst after using iron chloride, CAT-MM2: catalyst after using iron naphthenate, CAT-CD: catalyst after cyclic deactivation, E-cat: iron-contaminated equilibrium catalyst.

\subsection{Iron-Contaminated Catalysts' Morphology}

The surface morphology of an industrial iron-contaminated equilibrium catalyst is described elsewhere [25-27]. The iron nodules can be clearly observed on the E-cat surface in Figure 1a. During the formation of iron nodules, the iron formed a eutectic and released a lot of heat to melt the silica-alumina matrix layer [28]. A dense layer was formed that is typically a few microns in depth, as shown in Figure 2a.
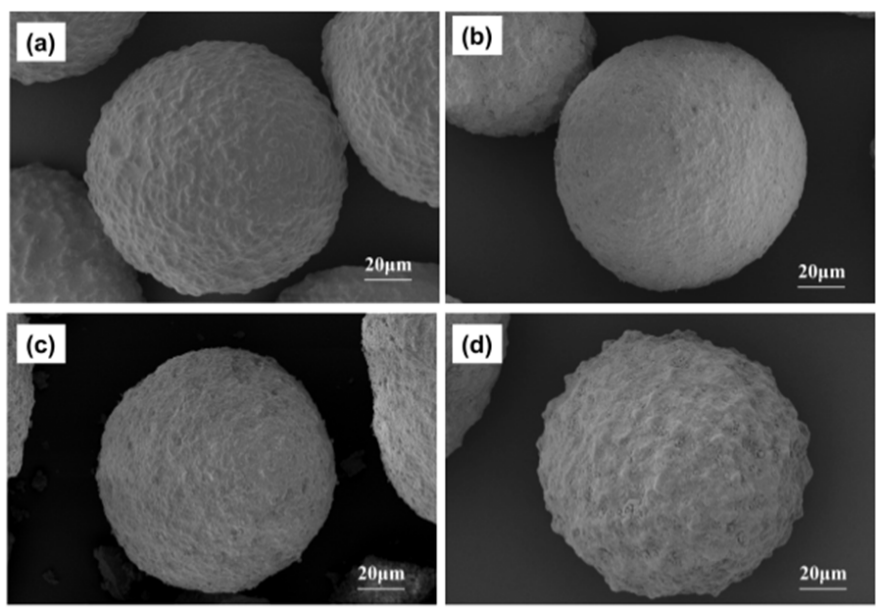

Figure 1. SEM micrographs of (a) E-cat, (b) CAT-MM1, (c) CAT-MM2, and (d) CAT-CD.
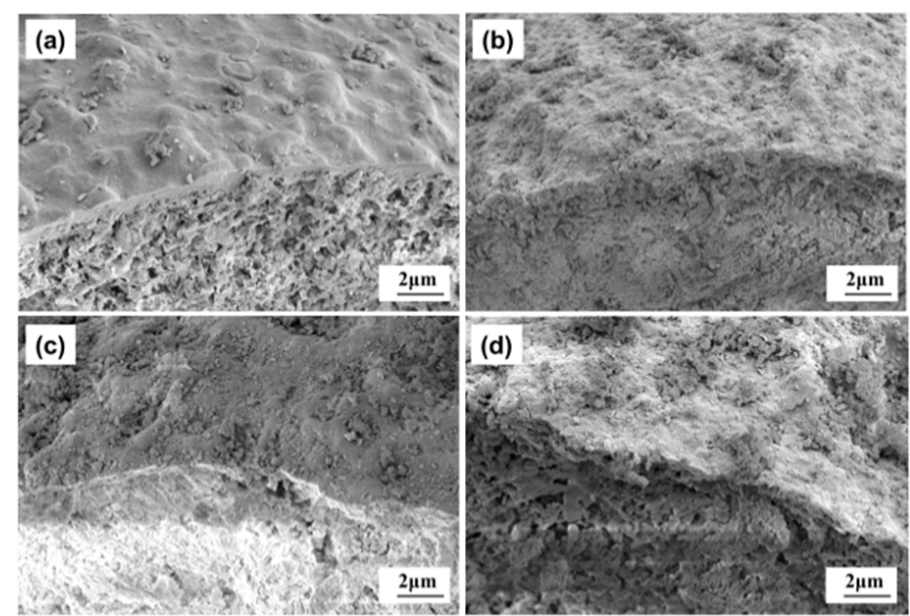

Figure 2. SEM micrographs of the catalyst cross-section: (a) E-cat, (b) CAT-MM1, (c) CAT-MM2, and (d) CAT-CD. 
The surfaces of CAT-MM1 and CAT-MM2 in Figure 1b,c were relatively smooth. The contamination sources used for the impregnation of CAT-MM1 and CAT-MM2 were iron chloride and iron naphthenate, where their lengths [25] were less than the pore diameter of CAT-BASE. During the impregnation process, iron chloride and iron naphthenate may have entered into the pore of the catalyst; therefore, no obvious iron nodules and boundary can be observed on the catalyst surface and cross-section (Figure 2b,c)

Iron nodules similar to those in Figure 1a can be observed on the surface of CAT-CD in Figure 1d. A few microns of the layer can also be observed in Figure 2d. Therefore, compared with the Mitchell impregnation method, the cyclic deactivation method could truly simulate the surface morphology of an industrial iron-contaminated equilibrium catalyst.

\subsection{Iron Distribution on the Catalyst}

Within the industrial FCC unit, the metals are usually deposited on the equilibrium catalyst with different deposition profiles. For example, nickel is known to deposit on the outer part of the catalyst [29]. Vanadium mainly diffuses from the outside to the inside $[30,31]$. Iron does not migrate to the interior of the catalyst but exists in the depth of 1 5 $\mu \mathrm{m}[32,33]$. Because the type of metal deposition dictates the activity and behavior of the metal, which may have an influence on the physical and chemical properties of the catalyst, the deposition characteristics are important to study and quantify [34].

As shown in Figure 3, all catalysts were line scanned from center to edge. The distribution of iron on E-cat gradually decreased from the outside to the inside in Figure $4 \mathrm{a}$. The iron mainly deposited on the outer surface of E-cat. In Figure $4 b, c$, the iron presented a uniform distribution from the center to the edge. During the process of the Mitchell impregnation, as the diameters of the iron chloride and iron naphthenate were smaller than that of the catalyst's pores, two kinds of iron-contaminated solution diffused into the catalyst pore at one time to form an equal-volume state. After calcination at a high temperature, the iron was evenly distributed in the catalyst. When using the Mitchell impregnation method to simulate iron contamination, due to the characteristics of the method, it is impossible to simulate the real reaction and regeneration environment of iron in an industrial plant. Therefore, iron was evenly distributed in the catalyst obtained using the Mitchell impregnation method.
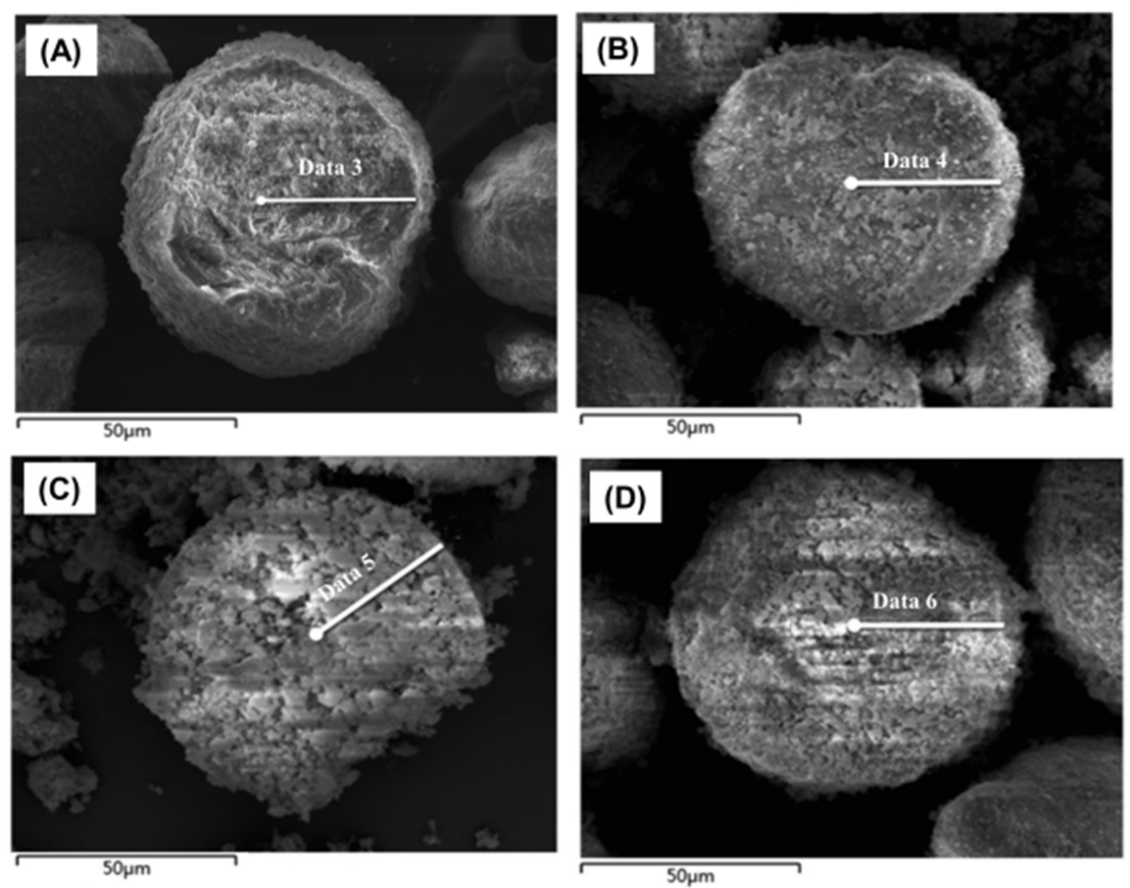

Figure 3. Line scan across a particle: (A) E-cat, (B) CAT-MM1, (C) CAT-MM2, and (D) CAT-CD. 

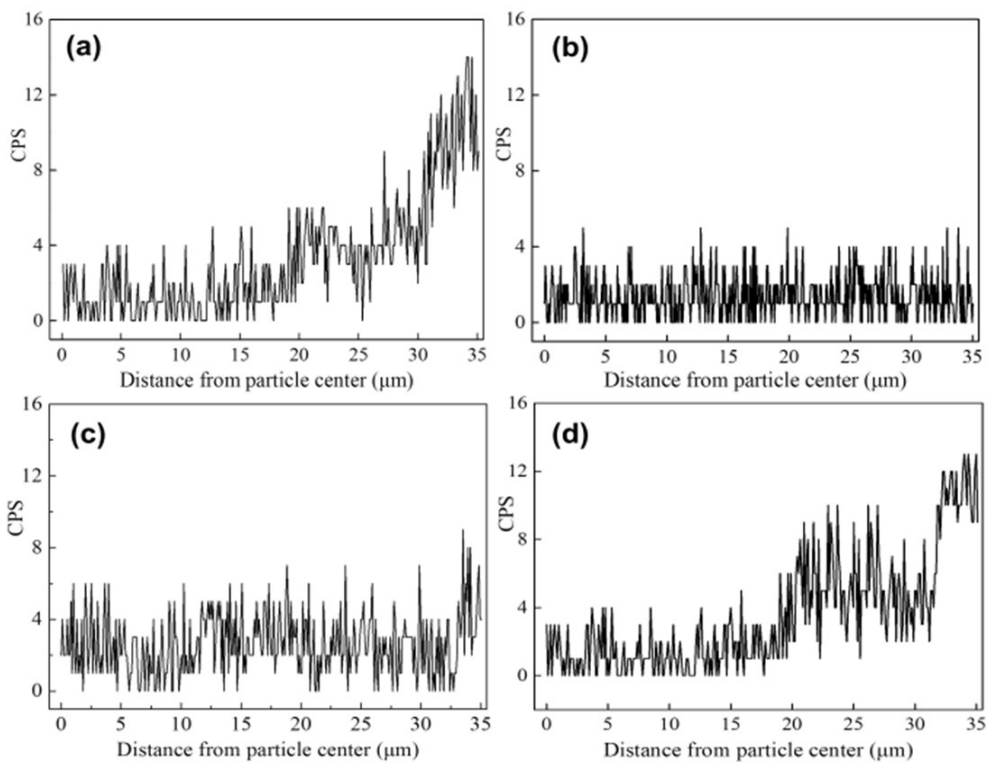

Figure 4. Iron distribution in a particle: (a) E-cat, (b) CAT-MM1, (c) CAT-MM2, and (d) CAT-CD. CPS: Counts Per Second.

The iron content in the depth of $1 \sim 5 \mu \mathrm{m}$ from the edge on CAT-CD was higher. When moving closer to the center of the catalyst, the iron content decreased and tended to become balanced (Figure $4 \mathrm{~d}$ ). The distribution of iron on CAT-CD was similar to that of E-cat. The CD method used multiple cycles of reaction and regeneration steps to realize heavy metal deposition on the catalyst's surface. In a single cycle, $10 \mathrm{~g}$ of vacuum gas oil (VGO; Fe on $\mathrm{VGO}=860 \mu \mathrm{g} / \mathrm{g}$ ) was pumped into the quartz reactor to react with $150 \mathrm{~g}$ of the catalyst. Iron compounds were deposited on the catalyst surface after the cracking step. During the regeneration step, the iron compounds formed a eutectic with silica, alumina, and alkali metal compounds and released a lot of heat to melt the silica-alumina matrix layer to block and cover the pores of the catalyst. With an increase in the number of cycles, the pore closing became more serious. It was difficult for the newly deposited iron to penetrate the catalyst, which could only react and exist in the range of a few microns on the catalyst's surface. Therefore, the distribution characteristics of iron on an industrial equilibrium catalyst can be simulated by using the cyclic deactivation method.

\subsection{Specific Surface Area}

The trend of the zeolite, matrix, and total surface areas measured using nitrogen adsorption for E-cat, the Mitchell impregnated catalysts (with different iron precursors), and the cyclic-deactivated catalysts are shown in Figure 5.

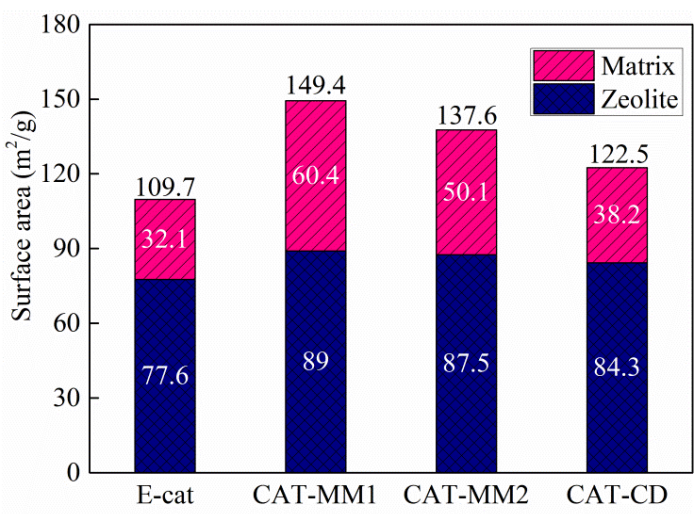

Figure 5. Zeolite, matrix, and total surface areas of the different catalysts. 
Among the three deactivation methods, the total surface area of CAT-CD was the closest to that of E-cat. The zeolite and matrix surface areas were also studied. The zeolite surface was not significantly affected by different deactivation methods, while the matrix surface area changed greatly. Because of the uniform distribution of iron on CAT-MM1 and CAT-MM2, the pore closing effect had no serious effect on the matrix surface area. As iron is mainly deposited on the outer surface of CAT-CD and forms a layer that is a few microns thick, its matrix surface area was the lowest among the three methods. The reduction in the total surface area caused by the iron contamination was mainly due to the influence of the matrix. Therefore, an iron resistance catalyst may be designed from the perspective of improving the ability of the matrix to accommodate iron.

\subsection{Iron Effects on the Catalyst Product Yields}

The product yields of catalysts contaminated using the Mitchell impregnation and cyclic deactivation methods were measured using an ACE unit. The product yield curves were obtained by changing the catalyst-to-oil ratio (C/O) [35]. The conversion curves as a function of the catalyst-to-oil ratio for different catalysts are shown in Figure 6.

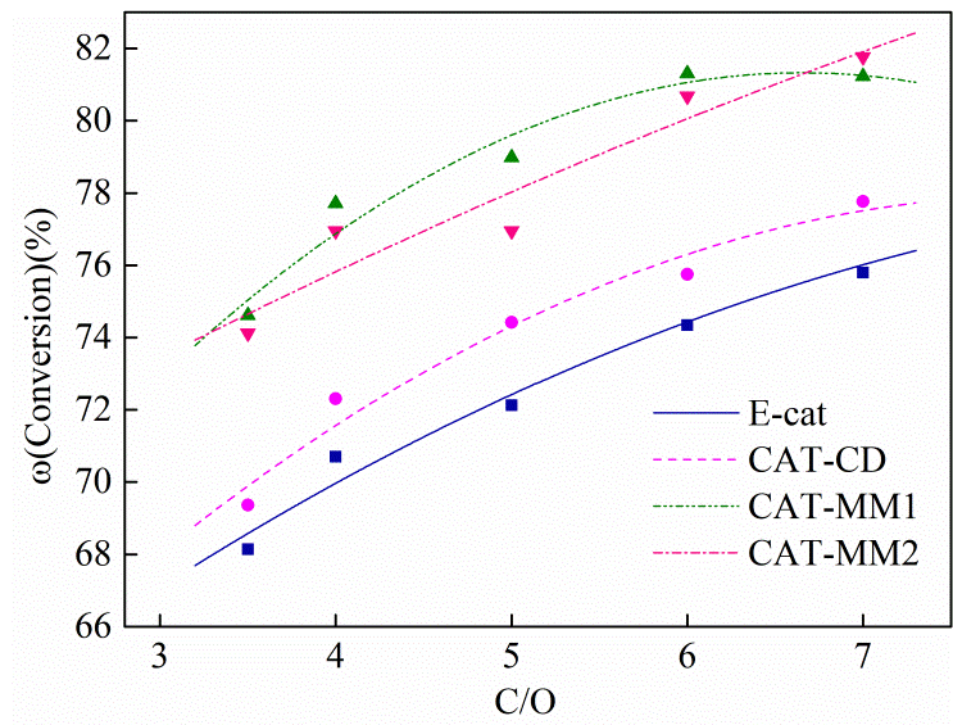

Figure 6. Conversion as a function of the catalyst-to-oil $(\mathrm{C} / \mathrm{O})$ ratio for different catalysts.

The catalysts' conversion increased with the increase of the $\mathrm{C} / \mathrm{O}$ ratio. The adjustment of the $\mathrm{C} / \mathrm{O}$ ratio could be realized by changing the catalysts' addition amounts under a fixed feed injection quantity. An increased $\mathrm{C} / \mathrm{O}$ ratio meant that more catalysts were added and more active centers were able to function in the reaction, which eventually led to the increase in conversion.

The average conversion of the catalyst that was deactivated using the $C D$ method was lower than that using the Mitchell impregnation method. The conversion values and trends of CAT-CD were also close to those of E-cat. During the process of iron contamination using the Mitchell impregnation method, the effect of pore closing on the catalyst was not serious, which led to the highest conversions for CAT-MM1 and CAT-MM2. A layer that was a few microns thick covered the surface of CAT-CD, which prevented the accessibility of the active sites of the catalyst may have resulted in an at least $4.84 \%$ decrease compared to CAT-MM1 and CAT-MM2.

The hydrogen factor curves of different catalysts are shown in Figure 7. The hydrogen factor is the mole ratio of hydrogen to methane in cracking products, which is an indicator of dehydrogenation reactions and can reflect the dehydrogenation ability of a catalyst [36]. 


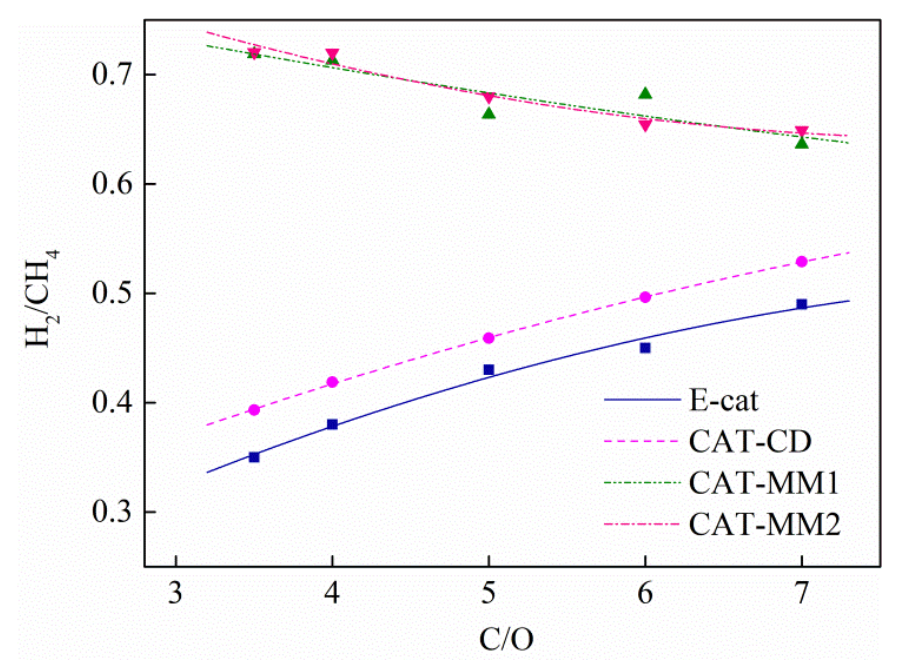

Figure 7. Hydrogen factor $\left(\mathrm{H}_{2} / \mathrm{CH}_{4}\right.$ ratio) as a function of the catalyst-to-oil ratio for different catalysts.

The dehydrogenation ability of E-cat was the weakest, as part of the iron was passivated after a long period of operation in the FCC unit. Both CAT-MM1 and CAT-MM2 had the strongest dehydrogenation ability and their hydrogen factor values were close. Iron penetrated through the inner channels of CAT-MM1 and CAT-MM2. Therefore, it had a stronger dehydrogenation capacity under the same contamination content. The hydrogen factor curves were in the highest position. During the process of cyclic deactivation, part of the iron may have gathered in the nodules and had a weak dehydrogenation capacity. Therefore, the dehydrogenation capacity of CAT-CD was lower than for CAT-MM1 and CAT-MM2 under the same contamination content. CAT-CD could truly simulate the dehydrogenation capacity of an industrial iron-contaminated equilibrium catalyst.

The bottoms curves of the different catalysts are shown in Figure 8. According to the positions of the different curves, the bottoms yield of the catalyst that was deactivated using the Mitchell impregnation method was lower than that of E-cat. The matrix surface area of CAT-MM1 and CAT-MM2 was less affected. The feed molecules and products could move relatively freely in the catalyst channel. Therefore, the average bottoms yields of CAT-MM1 and CAT-MM2 were $4.4 \%$ lower than that of E-cat. The pore structure of CAT-CD was seriously affected by iron nodules such that its matrix surface area greatly decreased. It was difficult for the feed molecules to diffuse into the catalyst and contact the active center for cracking. As a result, the bottoms yield of CAT-CD greatly increased. Its bottoms curve was similar to that of E-cat.

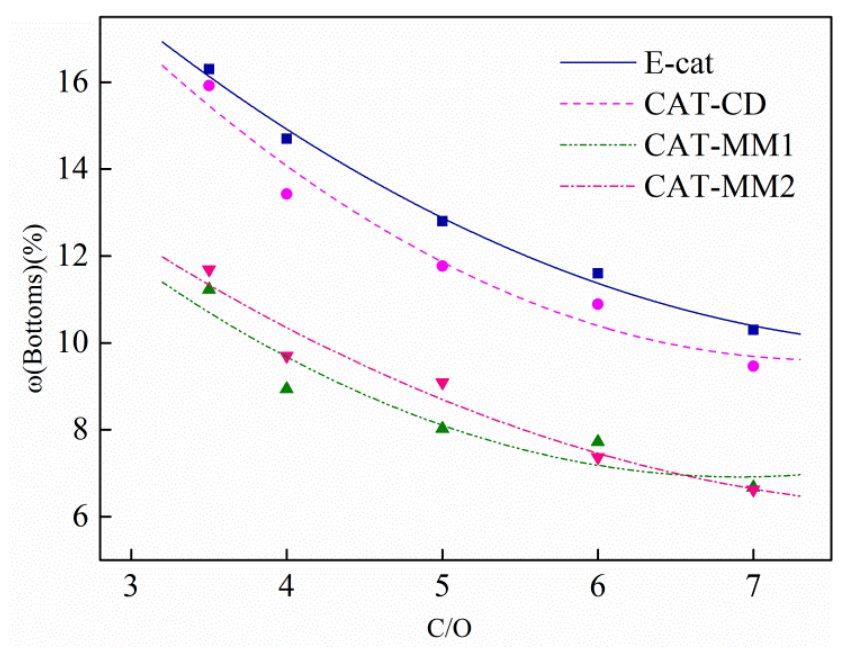

Figure 8. Bottoms as a function of the catalyst-to-oil ratio for different catalysts. 
The products' selectivities toward the catalysts that were deactivated using different methods are indicated in Table 2 at the same $74 \mathrm{wt} . \%$ conversion.

Table 2. Product yields at 74 wt.\% conversion.

\begin{tabular}{ccccc}
\hline $\boldsymbol{\omega}$ (Yields) $\mathbf{( \% )}$ & E-Cat & CAT-CD & CAT-MM1 & CAT-MM2 \\
\hline Coke & 9.18 & 9.33 & 10.29 & 9.87 \\
Dry gas & 3.28 & 3.22 & 3.24 & 3.06 \\
$\mathrm{H}_{2}$ & 0.46 & 0.51 & 0.69 & 0.67 \\
Gasoline & 44.56 & 44.87 & 43.00 & 43.19 \\
LCO & 14.06 & 13.89 & 14.21 & 14.12 \\
Bottoms & 11.94 & 12.11 & 11.79 & 11.88 \\
LPG & 16.88 & 16.58 & 17.47 & 17.88 \\
\hline
\end{tabular}

LCO: Light Cyclic Oil; LPG: Liquefied Petroleum Gas.

The coke and hydrogen selectivities of E-cat and CAT-CD were similar and were smaller than those of CAT-MM1 and CAT-MM2. This indicates that the iron on CATMM1 and CAT-MM2 had stronger dehydrogenation activity under the same amount of iron contamination, which produced more coke and hydrogen. Part of the iron in E-cat and CAT-CD was embedded and hence lost dehydrogenation activity, resulting in weak dehydrogenation activity. The bottoms selectivities of E-cat and CAT-CD were higher than those of CAT-MM1 and CAT-MM2, which indicates that the impregnated catalyst had a better cracking capacity for heavy oil. The product selectivity of CAT-CD was closer to that of E-cat.

\section{6. $\mathrm{NH}_{3}-\mathrm{TPD}$ Analysis of Different Catalysts}

Figure 9 shows the $\mathrm{NH}_{3}$-TPD (Temperature Programmed Desorption) profiles of E-cat and the catalysts prepared using different methods. The $\mathrm{NH}_{3}-\mathrm{TPD}$ profiles demonstrate that the peak area of the weak and medium-strong acids of CAT-CD was smaller than those of CAT-MM1 and CAT-MM2. A pore-closing effect made it harder for the $\mathrm{NH}_{3}$ molecules to get through the pores, which meant that the number of acid sites in CAT-CD was fewer. The number of acid sites was equal to the number of active sites. The number of active sites decreased in the following order: CAT-MM1 > CAT-MM2 > E-cat > CAT-CD. This was consistent with the change in the catalyst conversion and product distribution discussed before.

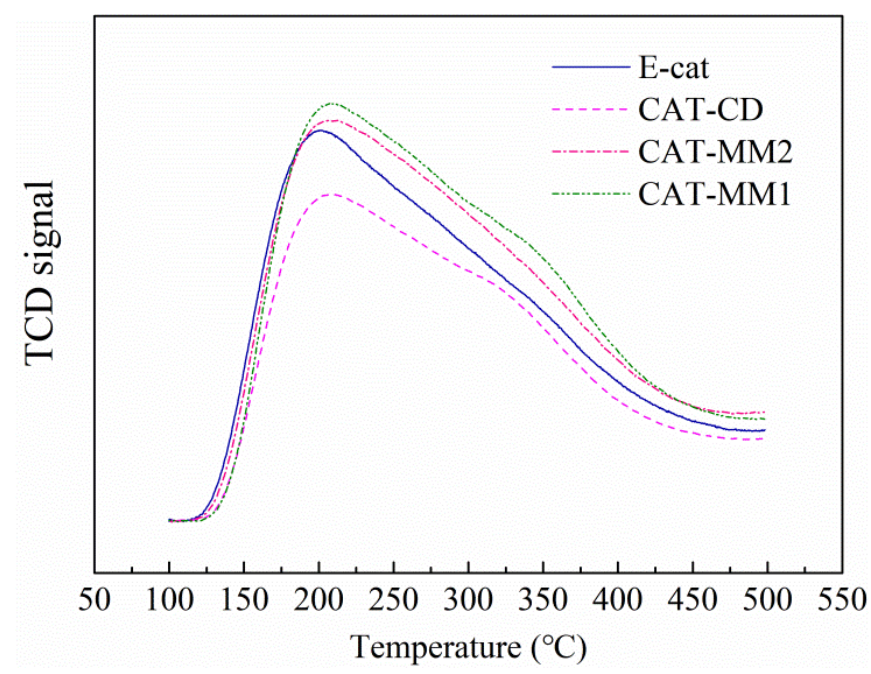

Figure 9. $\mathrm{NH}_{3}-\mathrm{TCD}$ (Thermal Conductivity Detector) profiles of the different catalysts. 


\subsection{SEM-EDS Analysis of the Iron Nodule Formation Mechanism}

The element content on the raised and valley areas of the catalyst deactivated using the cyclic deactivation method was observed in area scan mode using scanning electron microscopy (SEM) in combination with X-ray energy dispersive spectroscopy (EDS).

The distribution of silicon, aluminum, iron, calcium, and sodium in the selected area was analyzed using area scan mode. Six areas were selected as shown in Figure 10 for area scan analyze. The results are shown in Table 3.

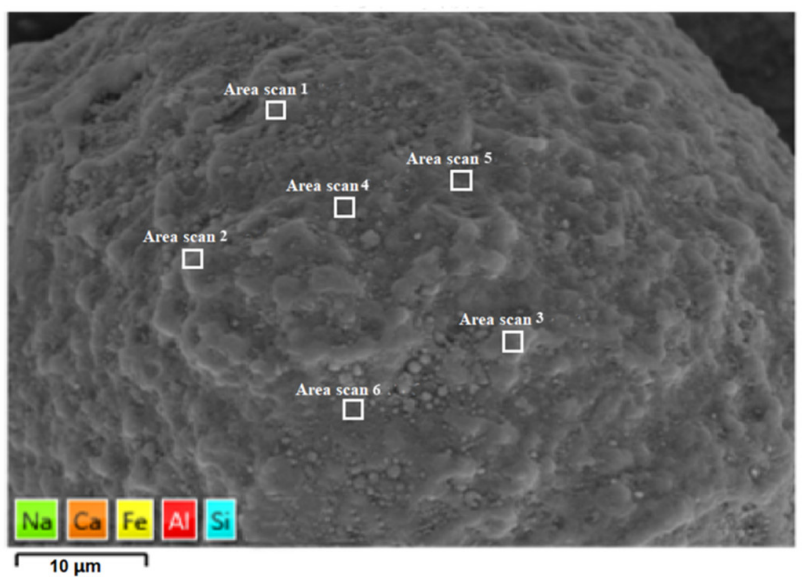

Figure 10. Area scan of a particle of CAT-CD.

Table 3. SEM-EDS analysis results of the element contents on CAT-CD.

\begin{tabular}{ccccccc}
\hline Location & Scanning Area & Si & Al & Fe & Na & Ca \\
\hline \multirow{2}{*}{ Raised area } & Area scan 1 (\%) & 34.16 & 48.89 & 15.18 & 1.77 & 0 \\
on surface & Area scan 2 (\%) & 33.55 & 43.62 & 18.29 & 4.53 & 0 \\
& Area scan 3 (\%) & 38.91 & 43.42 & 16.36 & 1.31 & 0 \\
\hline \multirow{2}{*}{ Valley area } & Area scan 4 (\%) & 45.94 & 40.94 & 11.86 & 1.26 & 0 \\
on surface & Area scan 5 (\%) & 43.91 & 42.69 & 11.59 & 1.81 & 0 \\
& Area scan 6 (\%) & 43.60 & 41.35 & 11.41 & 3.64 & 0 \\
\hline
\end{tabular}

The content of aluminum was higher than that of silicon in the raised area, and it was the opposite in the valley area. The initial melting temperatures of the eutectic that consisted of $\mathrm{Na}_{2} \mathrm{O}, \mathrm{Al}_{2} \mathrm{O}_{3}$, and $\mathrm{SiO}_{2}$ were higher than $770{ }^{\circ} \mathrm{C}$. It was difficult for $\mathrm{Fe}_{2} \mathrm{O}_{3}$ to combine with $\mathrm{Al}_{2} \mathrm{O}_{3}, \mathrm{Na}_{2} \mathrm{O}$, and $\mathrm{SiO}_{2}$ to form low-melting-temperature phases in areas that were rich in $\mathrm{Al}_{2} \mathrm{O}_{3}$; therefore, the structure of this area was preserved.

However, in the areas with a relatively high content of $\mathrm{SiO}_{2}$, it was easy for the $\mathrm{Fe}_{2} \mathrm{O}_{3}$ to form a eutectic with $\mathrm{Na}_{2} \mathrm{O}$ and $\mathrm{SiO}_{2}$, whose initial melting temperatures were lower than $500{ }^{\circ} \mathrm{C}$ [14]. During the regeneration process, the $\mathrm{SiO}_{2}$ enrichment zone further intensified the melting and sintering, resulting in a regional collapse and local depression. The retention of the surface structure in the $\mathrm{Al}_{2} \mathrm{O}_{3}$-rich areas and the collapse of the surface structure in the $\mathrm{SiO}_{2}$-rich areas resulted in a nodule morphology on the highly iron-contaminated catalyst surface.

The formation of iron nodules was the main reason for the various performances of the catalysts contaminated by iron. During the process of the Mitchell impregnation, the ironcontaminated solution filled the internal channels of the catalyst to form an equal-volume state. After the high-temperature calcining treatment, the iron was evenly distributed in the catalyst and there was no condition that allowed for the formation of iron nodules [37]. Therefore, iron nodules could not be observed on the catalyst's surface and the deactivated catalyst could not simulate an iron-contaminated equilibrium catalyst.

Iron deposition on the surface of the cyclic-deactivated catalyst was realized using long-time repeated cracking-regeneration cycles. The iron deposition in a single cycle was 
very low. After the cracking step, iron oxide combined with silicon oxide, aluminum oxide, and alkali metal oxides to form low-melting-temperature phases before it diffused inside the catalyst. The formation conditions of iron nodules in industrial plants could be simulated by using the cyclic deactivation method. The CD method is an effective and accurate method for simulating iron contamination in a laboratory.

\section{Materials and Methods}

\subsection{Materials}

In this research, a commercial catalyst (CAT-BASE) without iron contamination, which was used to simulate iron deactivation, was collected from the industrial plant of the Dalian West Pacific Petrochemical Co., Ltd. (WEPEC, Dalian, China). In order to simulate the actual state of an iron-contaminated equilibrium catalyst in an FCC unit, the target amount of iron deposition and appropriate conditions were selected to carry out Mitchell impregnation and the cyclic deactivation method. The equilibrium catalyst (E-cat) with a high level of iron contamination was also collected from WEPEC.

\subsection{Methods}

\subsubsection{Mitchell Impregnation}

Iron chloride and iron naphthenate were used as contamination species to carry out this method. Iron chloride was added to deionized water (Fe on solution $=14,400 \mu \mathrm{g} / \mathrm{g}$ ) and iron naphthenate was added to a toluene solution (Fe on solution $=12,500 \mu \mathrm{g} / \mathrm{g}$ ). The catalysts $(150 \mathrm{~g})$ were impregnated with $120 \mathrm{~g}$ of iron chloride solution and $150 \mathrm{~g}$ of iron naphthenate solution, respectively, dried for $2 \mathrm{~h}$ at $150{ }^{\circ} \mathrm{C}$, and then calcined for $6 \mathrm{~h}$ at $600{ }^{\circ} \mathrm{C}$ before use. During the drying process, the catalyst was stirred every $20 \mathrm{~min}$ to ensure that it was evenly heated and would not agglomerate. The catalysts that were impregnated with iron chloride and iron naphthenate were labeled as CAT-MM1 and CAT-MM2, respectively.

\subsubsection{Cyclic Deactivation}

The deactivated catalyst was treated using 200 cycles of cracking, stripping, regeneration, and cooling in the MCD (Multi Cyclic Deactivation) unit produced by $360^{\circ} \mathrm{KAS}$ Company (Amsterdam, The Netherlands). The cracking step was performed at a reaction temperature of $530{ }^{\circ} \mathrm{C}$ and the regeneration step was performed at $780^{\circ} \mathrm{C}$. During the cracking step, $10 \mathrm{~g}$ of VGO spiked with iron naphthenate (Fe on VGO $=860 \mu \mathrm{g} / \mathrm{g}$ ) was pumped into the quartz reactor to react with $150 \mathrm{~g}$ of the catalyst at the reaction temperature. Finally, the aged catalyst was marked as CAT-CD.

\subsubsection{Catalyst Characterization}

The metal content was determined using a ZSX PrimusIIX-ray fluorescence instrument produced by the Japan Science and Technology Company (Tokyo, Japan). The X-ray tube voltage was $50 \mathrm{kV}$, the tube current was $50 \mathrm{~mA}$, and the diaphragm aperture was $20 \mathrm{~mm}$.

The catalyst phase structure was determined using a D/max-3C X-ray diffractometer produced by the Japan Science and Technology Company (Tokyo) with the following settings: $\mathrm{Cu}$ target, $\mathrm{K} \alpha$ radiation, tube voltage: $40 \mathrm{kV}$, tube current: $20 \mathrm{~mA}$, scanning range: 5 to $50^{\circ}$, scanning speed: $4^{\circ} / \mathrm{min}$.

$\mathrm{NH}_{3}$-TPD was performed on a Micromeritics AUTOCHEM II 2920 produced by the Micromeritics Instruments Corporation (Norcross, GA, USA). $0.1 \mathrm{~g}$ of sample was purified with $\mathrm{He}$ gas flow at room temperature for $1 \mathrm{~h}$. $\mathrm{NH}_{3}$ adsorption was performed by adjusting temperature with two-stage method. The desorption curve was obtained after the adsorption is stable, and the temperature was raised to $500{ }^{\circ} \mathrm{C}$ within $50 \mathrm{~min}$.

The specific surface area and pore volume of the catalyst were measured using a Micromeritics ASAP 3000 automatic physical adsorption instrument produced by the Micromeritics Instruments Corporation (Norcross, GA, USA). The surface area was deduced from the adsorption isotherms using the BET (Brunauer, Emmett and Teller) equation. 
The catalyst surface morphology was observed using an ULTRA PLUS thermal field emission scanning electron microscope produced by Zeiss Optical Instruments (Oberkochen, Germany). Secondary electron resolution: $1.0 \mathrm{~nm}(15 \mathrm{kV})$ and $1.9 \mathrm{~nm}(1 \mathrm{kV})$; electron gun: LaB6 thermal field emission electron gun; acceleration voltage: $0.1 \sim 30 \mathrm{kV}$, magnification: $\times 12 \sim \times 10000$.

The iron distribution on the catalysts' cross-section was analyzed using line scan mode during SEM in combination with an X-ray energy dispersive spectroscope produced by Oxford Instruments (Abingdon, UK). Resolution: better than $127 \mathrm{eV}$; The MnK $\alpha$ peak-toback ratio: 20000:1; stability: 1000 100,000 cps, spectral peak drift $<1 \mathrm{eV}$, resolution change $<1 \mathrm{eV}$. All four catalysts were measured from the center to the edge. In order to ensure the accuracy of the measurement, a total of 350 points were measured by using a spot analysis technique over a length of $35 \mu \mathrm{m}$ to make up the results of the line scan.

\subsubsection{Catalyst Cracking Activity and Selectivity}

The cracking performance of different catalysts was tested in an Advanced Cracking Evaluation unit developed by the KTI Technology Company (Huston, TX, USA). The flowchart of the ACE unit is shown in Figure 11. The reaction conditions were the following: The amount of catalyst added was $9 \mathrm{~g}$. The reaction and regeneration temperatures were $530{ }^{\circ} \mathrm{C}$ and $715^{\circ} \mathrm{C}$, respectively. The variation of the catalyst-to-oil ratio $(\mathrm{C} / \mathrm{O}=3.5,4.0,5.0,6.0,7.0)$ was performed by varying the catalyst amount. Gaseous products were analyzed using an GC-3000 on-line chromatograph produced by INFICON Company (East Syracuse, NY, USA) according to the UOP method 539. GC-3000 uses four chromatographic modules for detection, the work temperature is $0{ }^{\circ} \mathrm{C} \sim 50{ }^{\circ} \mathrm{C}$. The carrier gases used are helium, hydrogen, nitrogen and argon. The simulated distillation of liquid products was carried out using a 7890B chromatograph produced by Agilent Technologies Inc. (Santa Clara, CA, USA) according to the SH/T 0558 procedure. The working environmental temperature of Agilent 7890B is $15^{\circ} \mathrm{C} \sim 35$. Retention time repeatability $<0.0008 \mathrm{~min}$. Carrier and makeup gas settings selectable for helium, hydrogen, nitrogen and argon/methane. Coke deposited on the catalyst was quantified using a $\mathrm{CO}_{2}$ analyzer produced by Servomex Group Co., Ltd (Sussex, UK). The detection range of $\mathrm{CO}_{2}$ analyzer is $0 \sim 20 \%$. When the detection value is lower than $0.4 \%$, the regeneration step is considered to be completed. Conversion and yields of dry gas $\left(\mathrm{H}_{2}+\mathrm{C} 1+\mathrm{C} 2\right)$, LPG (Liquefied Petroleum Gas) $(\mathrm{C} 3+\mathrm{C} 4)$, gasoline $\left(\mathrm{C} 5<\mathrm{bp}<221^{\circ} \mathrm{C}\right), \mathrm{LCO}\left(\right.$ Light Cyclic Oil) $\left(221^{\circ} \mathrm{C}<\mathrm{bp}<343^{\circ} \mathrm{C}\right)$, $\mathrm{HCO}$ (Heavy Cyclic Oil) $\left(\mathrm{bp}>343^{\circ} \mathrm{C}\right)$, and coke were calculated.

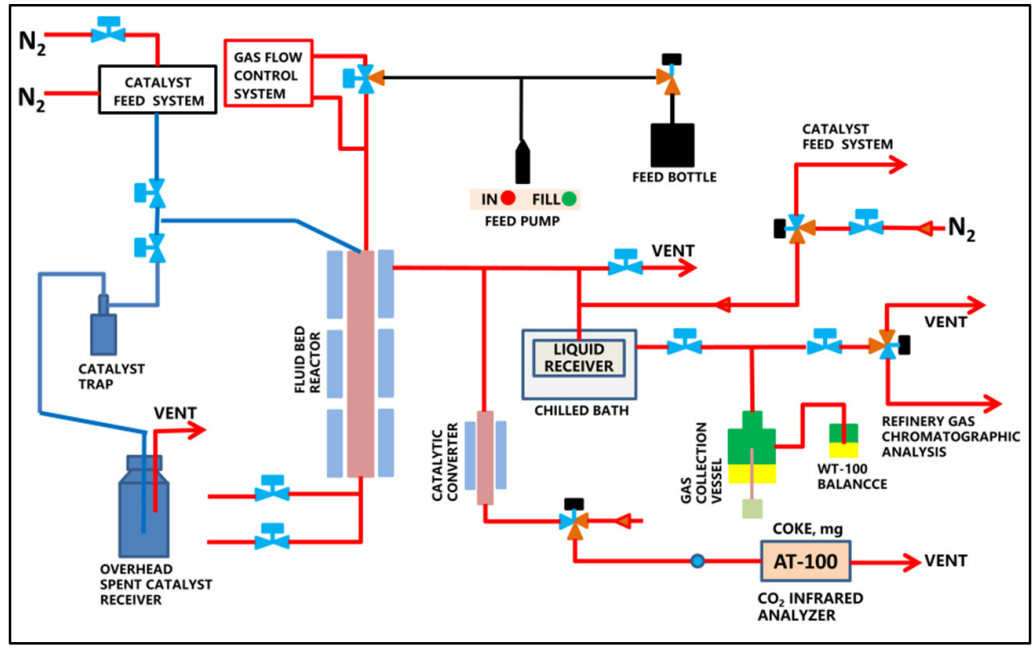

Figure 11. The flowchart of the Advanced Cracking Evaluation (ACE) unit. 


\section{Conclusions}

Iron nodules are the main factor that leads to the poor performance of iron-contaminated catalysts. The retention of the surface structure in the alumina-rich areas and the collapse of the surface structure in the silica-rich areas resulted in a continuous nodule morphology on a highly iron-contaminated equilibrium catalyst.

Iron was evenly distributed in the catalyst prepared using the Mitchell impregnation method, and no obvious iron nodules or boundary were found on the surface of the catalyst. The surface area was less affected by iron contamination. Iron deposited on the catalyst resulted in a small reduction in the conversion and it had little effect on the bottoms selectivity, but it led to a strong dehydrogenation capacity. The catalytic performance of the impregnated catalyst was quite different from that of the iron-contaminated equilibrium catalyst.

The iron was mainly present in the depth of $1 \sim 5 \mu \mathrm{m}$ from the edge on the catalyst prepared using the cycle deactivation method. As iron nodules formed on the surface of the cyclic-deactivated catalyst, pore closing limited the feed molecules' diffusion inside the particles and the products' diffusion outward, resulting in a significant decrease in conversion, extremely poor bottoms selectivity, and a small increase in dehydrogenation capacity.

We established a laboratory simulation method that can accurately simulate the industrial iron-contaminated equilibrium catalyst. By using this method, we can recognize the real mechanism of iron contamination, understand the main factors that affect the physical and chemical properties of an iron-contaminated catalyst, and predict the actual reaction performance and product distribution of a catalyst prepared in a laboratory. This can effectively reduce the time and economic costs caused by the failure of laboratory simulations.

In our next research work, we can rely on the accurate $\mathrm{CD}$ method to develop iron tolerance technology. On the other hand, we can also investigate the combined effect of iron and other heavy metals, such as nickel and vanadium, on the catalyst to further improve the iron deactivation simulation method.

Author Contributions: Y.L. and T.L. conceived and designed the experiment; Y.L. performed the experiments and analyzed the data. All the authors listed have made contributions to drafting and writing the manuscript. All authors have read and agreed to the published version of the manuscript.

Funding: This research was funded by the Ministry of Science and Technology Management of PetroChina (2019D-5006-20).

Institutional Review Board Statement: Not applicable.

Informed Consent Statement: Not applicable.

Data Availability Statement: Data available in a publicly accessible repository.

Acknowledgments: We acknowledge the financial support from the Development of New Catalysts with Low Emission and High Gasoline Yield project, the Ministry of Science and Technology Management of PetroChina (2019D-5006-20). We thank L. Zhang for kind assistance in SEM-EDS experiments, and X.Q. Sun for her kind helps in $\mathrm{NH}_{3}$-TPD analysis.

Conflicts of Interest: The authors declare no conflict of interest.

\section{References}

1. Rainer, D.R.; Rautiainen, E.; Imhof, P. Novel lab-scale deactivation method for FCC catalyst: Inducing realistic accessibility responses to iron poisoning. Appl. Catal. A Gen. 2003, 249, 69-80. [CrossRef]

2. Vincz, C.; Rath, R.; Smith, G.M.; Yilmaz, B.; Mcguire, R. Dendritic nickel porphyrin for mimicking deposition of contaminant nickel on FCC catalysts. Appl. Catal. A Gen. 2015, 495, 39-44. [CrossRef]

3. Mathieu, Y.; Corma, A.; EchardI, M.; Bories, M. Single and combined fluidized catalytic cracking (FCC) catalyst deactivation by iron and calcium metal-organic contaminants. Appl. Catal. A Gen. 2014, 469, 451-465. [CrossRef]

4. Mehla, S.; Kukade, S.; Kumar, P.; Rao, P.V.C.; Sriganesh, G.; Ravishankar, R. Fine tuning h-transfer and $\beta$-scission reactions in VGO FCC using metal promoted dual functional ZSM-5. Fuel 2019, 242, 487-495. [CrossRef] 
5. Tangstad, E.; Andersen, A.; Myhrvold, E.M.; Myrstad, T. Catalytic behaviour of nickel and iron metal contaminants of an FCC catalyst after oxidative and reductive thermal treatments. Appl. Catal. A Gen. 2008, 346, 194-199. [CrossRef]

6. Zhang, C.C.; Gao, X.; Yilmaz, B. Development of FTIR Spectroscopy Methodology for Characterization of Boron Species in FCC Catalysts. Catalysts 2020, 10, 1327. [CrossRef]

7. Souza, N.L.A.; Tkach, I.; Morgado, E., Jr.; Krambrock, K. Vanadium poisoning of FCC catalysts: A quantitative analysis of impregnated and real equilibrium catalysts. Appl. Catal. A. Gen. 2018, 560, 206-214. [CrossRef]

8. Long, J.; Zhu, Y.X.; Liu, Y.J.; Da, Z.J.; Zhou, H. Effects of vanadium oxidation number on desulfurization performance of FCC catalyst. Appl. Catal. A Genera 2005, 282, 295-301. [CrossRef]

9. Whitcombe, J.M.; Agranovski, I.E.; Braddock, R.D. Identification of Metal Contaminants on FCC Catalyst. Part. Part. Syst. Charact. 2005, 22, 268-275. [CrossRef]

10. Tian, H.; Huang, C.; Fan, Z. Metals on a novel USY zeolite after hydrothermal aging. Stud. Surf. Sci. Catal. 2001, 139, S0167-S2991.

11. Sandoval-Díaz, L.; Ruíz-Cardona, Y.; Trujillo, C. Amorphization of USY zeolite induced by sodium chloride and high temperature steaming. Microporous Mesoporous Mater. 2016, 224, 168-175. [CrossRef]

12. Liu, Z.; Zhang, Z.; Liu, P.; Zhai, J.; Yang, C. Iron contamination mechanism and reaction performance research on FCC catalyst. J. Nanotechnol. 2015, 2015. [CrossRef]

13. Whitcombe, J.M.; Agranovski, I.E.; Braddock, R.D. Impact of metal ridging on the fluidization characteristics of FCC catalyst. Chem. Eng. Technol. 2002, 25, 981-987. [CrossRef]

14. Yaluris, G.; Cheng, W.C.; Peters, M.; Mcdowell, L.T.; Hunt, L. Mechanism of fluid cracking catalysts deactivation by Fe. Stud. Surf. Sci. Catal. 2004, 149, 139-163. [CrossRef]

15. Buurmans, I.L.; Soulimani, F.; Ruiz-Martínez, J.; van der Bij, H.E.; Weckhuysen, B.M. Structure and acidity of individual fluid catalytic cracking catalyst particles studied by synchrotron-based infrared micro-spectroscopy. Microporous Mesoporous Mater. 2013, 166, 86-92. [CrossRef]

16. Nguyen, T.H.; Ngo, P.T.; Tran, T.V.; Nguyen, S.; Vu, D.M.; Ha, Q.L.M.; Dao, X.T.T.; Dang, T.T. Effect of hydrothermal conditions on the catalytic deactivation of a fluid cracking catalyst. React. Kinet. Mech. Catal. 2013, 109, 563-574. [CrossRef]

17. Etim, U.J.; Xu, B.; Bai, P.; Ullah, R.; Yan, Z. Role of nickel on vanadium poisoned FCC catalyst: A study of physiochemical properties. J. Energy Chem. 2016, 25, 667-676. [CrossRef]

18. Etim, U.J.; Xu, B.; Ullah, R.; Yan, Z. Effect of vanadium contamination on the framework and micropore structure of ultra stable Y-zeolite. J. Colloid Interface Sci. 2016, 463, 188-198. [CrossRef]

19. Chiranjeevi, T.; Gokak, D.T.; Ravikumar, V.; Viswanathan, P.S. Development of new deactivation method for simulation of fluid catalytic cracking equilibrium catalyst. J. Chem. Sci. 2014, 126, 353-360. [CrossRef]

20. Wallenstein, D.; Farmer, D.; Knoell, J.; Fougret, C.M.; Brandt, S. Progress in the deactivation of metals contaminated FCC catalysts by a novel catalyst metallation method. Appl. Catal. A. Gen. 2013, 462, 91-99. [CrossRef]

21. Psarras, A.C.; Iliopoulou, E.F.; Nalbandian, L.; Lappas, A.A.; Pouwels, C. Study of the accessibility effect on the irreversible deactivation of FCC catalysts from contaminant feed metals. Catal. Today 2007, 127, 44-53. [CrossRef]

22. Almas, Q.; Naeem, M.A.; Baldanza, M.A.S.; Solomon, J.; Kenvin, J.C.; Christoph, R.; Müller, C.R.; da Silva Teixeira, V.; Jones, C.W.; Sievers, C. Transformations of FCC catalysts and carbonaceous deposits during repeated reaction-regeneration cycles. Catal. Technol. 2019, 9, 6977-6992. [CrossRef]

23. Rainer, D.R.; Rautiainen, E.; Nelissen, B.; Imhof, P.; Vadovic, C. Simulating iron-induced FCC accessibility losses in lab-scale deactivation. Stud. Surf. Sci. Catal. 2004, 149, 165-176. [CrossRef]

24. Brandt, S.; Knöll, J.; Jiang, H.; Saar, J.; Fougret, C. The role of magnetic susceptibility in detecting iron poisoning in FCC equilibrium catalyst samples and its combination with other macroscopic bulk analysis techniques. Ind. Eng. Chem. Res. 2019, 58, 20528-20535. [CrossRef]

25. Zhu, Y.X.; Du, Q.S.; Lin, W.; Tang, L.W.; Jun, L. Chapter 13 studies of iron effects on fcc catalysts. Stud. Surf. Sci. Catal. 2007, 166, 201-212. [CrossRef]

26. Jiang, H.; Livi, K.J.; Kundu, S.; Cheng, W.C. Characterization of iron contamination on equilibrium fluid catalytic cracking catalyst particles. J. Catal. 2018, 361, 126-134. [CrossRef]

27. Souza, N.L.; Paniago, R.; Ardisson, J.D.; Morgado, E., Jr.; Krambrock, K. Iron contamination of FCC catalysts: Quantification of different crystalline phases and valence states. Appl. Catal. A Gen. 2019, 569, 57-65. [CrossRef]

28. Hochheiser, T.; Tang, Y.; Allahverdi, M.; Graaf, B.D. FCC Additive Improves Residue Processing Economics with High Iron Feeds In Proceedings of the AFPM Annual Meeting, Orlando, FL, USA, 23-25 March 2014.

29. Meirer, F.; Morris, D.T.; Kalirai, S.; Liu, Y.; Andrews, J.C.; Weckhuysen, B.M. Mapping metals incorporation of a whole single catalyst particle using element specific X-ray nanotomography. J. Am. Chem. Soc. 2015, 137, 102-105. [CrossRef] [PubMed]

30. Pan, S.S.; Lin, L.T.X.; Komvokis, V.; Spann, A.; Clough, M.; Yilmaz, B. Nanomaterials Fueling the World. In Nanomaterials for Sustainable Energy; American Chemical Society: Washington, DC, USA, 2015; Volume 1213, pp. 3-18. [CrossRef]

31. Cristiano-Torres, D.V.; Osorio-Pérez, Y.; Palomeque-Forero, L.A.; Sandoval-Díaz, L.E.; Trujillo, C.A. The action of vanadium over Y zeolite in oxidant and dry atmosphere. Appl. Catal. A. Gen. 2008, 346, 104-111. [CrossRef]

32. Wise, A.M.; Weker, J.N.; Kalirai, S.; Farmand, M.; Shapiro, D.A.; Meirer, F.; Weckhuysen, B.M. Nanoscale chemical imaging of an individual catalyst particle with soft x-ray ptychography. Acs Catal. 2016, 6, 2178-2181. [CrossRef] [PubMed] 
33. Ihli, J.; Ferreira Sanchez, D.; Rosh, J.; Cuartero, V.; Mathon, O.; Krumeich, F.; Borca, C.; Huthwelker, T.; Cheng, W.-C.; Shu, Y.Y.; et al. Localization and speciation of iron impurities within a fluid catalytic cracking catalyst. Angew. Chem. 2017, 129, 14219-14223. [CrossRef]

34. Senter, M.; Mastry, M.C.; Mannion, A.M.; McGuire, R.; Houtz, D.; Yilmaz, B. Quantitative visual characterization of contaminant metals and their mobility in fluid catalytic cracking catalysts. Catalysts 2019, 9, 831. [CrossRef]

35. Alkhlel, A.; de Lasa, H. Catalyst/Feedstock Ratio Effect on FCC Using Different Catalysts Samples. Catalysts $2019,9,542$. [CrossRef]

36. Chen, J.W.; Xu, Y.H. Fluid Catalytic Cracking Process and Engineering; Sinopec Press: Beijing, China, 2018; pp. $392-395$.

37. Bai, P.; Etim, U.J.; Yan, Z.; Mintova, S.; Zhang, Z.; Zhong, Z.; Gao, X. Fluid catalytic cracking technology: Current status and recent discoveries on catalyst contamination. Catal. Rev. 2019, 61, 333-405. [CrossRef] 